\title{
Analgesia Nociception Index is More Sensitive Than the Bispectral Index In Pain Stimulation
}

Yi-Fang Chiu', Shou-Zen Fan'1, Jiann-Shing Shieh², Chih-Jun Lai', Jia-Rong Yeh³, Kuo-Kuang Jen ${ }^{4}$

1.Department of Anesthesiology, National Taiwan University Hospital

2.Department of Mechanical Engineering, Yuan Ze University, Taiwan

3.Research Center for Adaptive Data Analysis, National Central University, Taiwan

4. National Chung-Shan institute of science \& Technology (Missile \& Rocket Systems Research Division)

\section{Background and goal of study}

Anesthetic depth is an obscure term and difficult to define. Conventionally, the bispectral index (BIS), which is used for monitoring the depth of anesthesia, cannot be used to observe pain stimulation or detect cortex activity. The analgesia nociception index (ANI) is for monitoring changes in pain in the autonomic nerve system. ${ }^{1-2}$

We compared the sensitivity of the ANI and BIS during surgery while pain from sources such as intubation or incision was stimulated .

\section{Materials and methods}

Sixty- patients received scheduled surgery with ASA of I or II and anesthetic within $120 \mathrm{~min}$. The patients were aged 20-80 years and all had a BMI below 30. After receiving general anesthesia, the patients underwent BIS and ANI monitoring to allow us to observe the variance at 3 time points. The 3 time points were: 'under intubation' from $1 \mathrm{~min}$ before intubation to $2 \mathrm{~min}$ after intubation), 'after intubation' (from 2 min after intubation until incision) and 'Surgery 3 minutes' (after incision 3 minutes). ANI values in the range of $0-100$ points (lower values indicate higher levels of pain) and BIS values in the range of 0-100 points (higher values indicate a considerable higher level of consciousness) were recorded simultaneously.

\section{Results and Discussion}

Twenty-eight patients received intubation, 24 received supraglottic airway devices (SADs), and 8 received intravenous anesthesia (IVG). The "after intubation" condition was used as a control because there was no stimulation was present at this time point; "under intubation" and "Sugery 3 minutes" were operationalized as experimental groups. ANI values were lower in the "under intubation" and "Surgery 3 minutes" conditions than in the "after intubation" for both the intubation and SAD groups (Figs. 1 and 2). However, the BIS values were unchanged in both the "under intubation" and "Surgery into 3 minutes" conditions. Unexpectedly, in the IVG group (Fig. 3), the median ANI value at "Surgery in 3 minutes" was higher than that measured before surgery (no pain stimulation).

\section{Conclusion}

The ANI was more sensitive to pain stimulation than was the BIS. Of particular interest, the IVG group ANI values were higher at "Sugery into 3 minutes" than they were "before surgery.". The reasons for this could be the short duration of surgery, but this hypothesis requires further surveying for confirmation.

Figure 1. ANI/BIS Index in Intubation Group

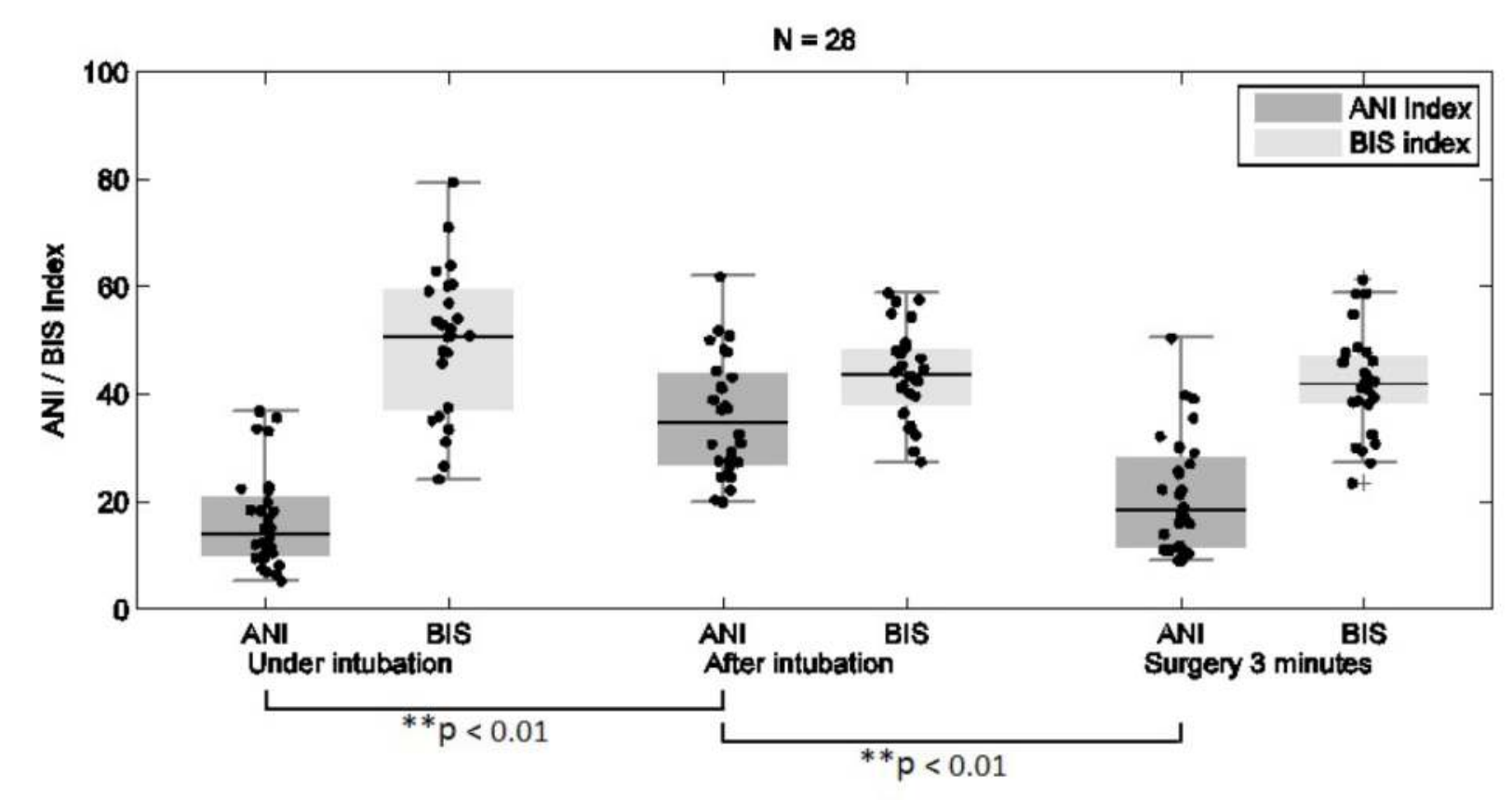

Figure 2. ANI/BIS Index in SAD Group

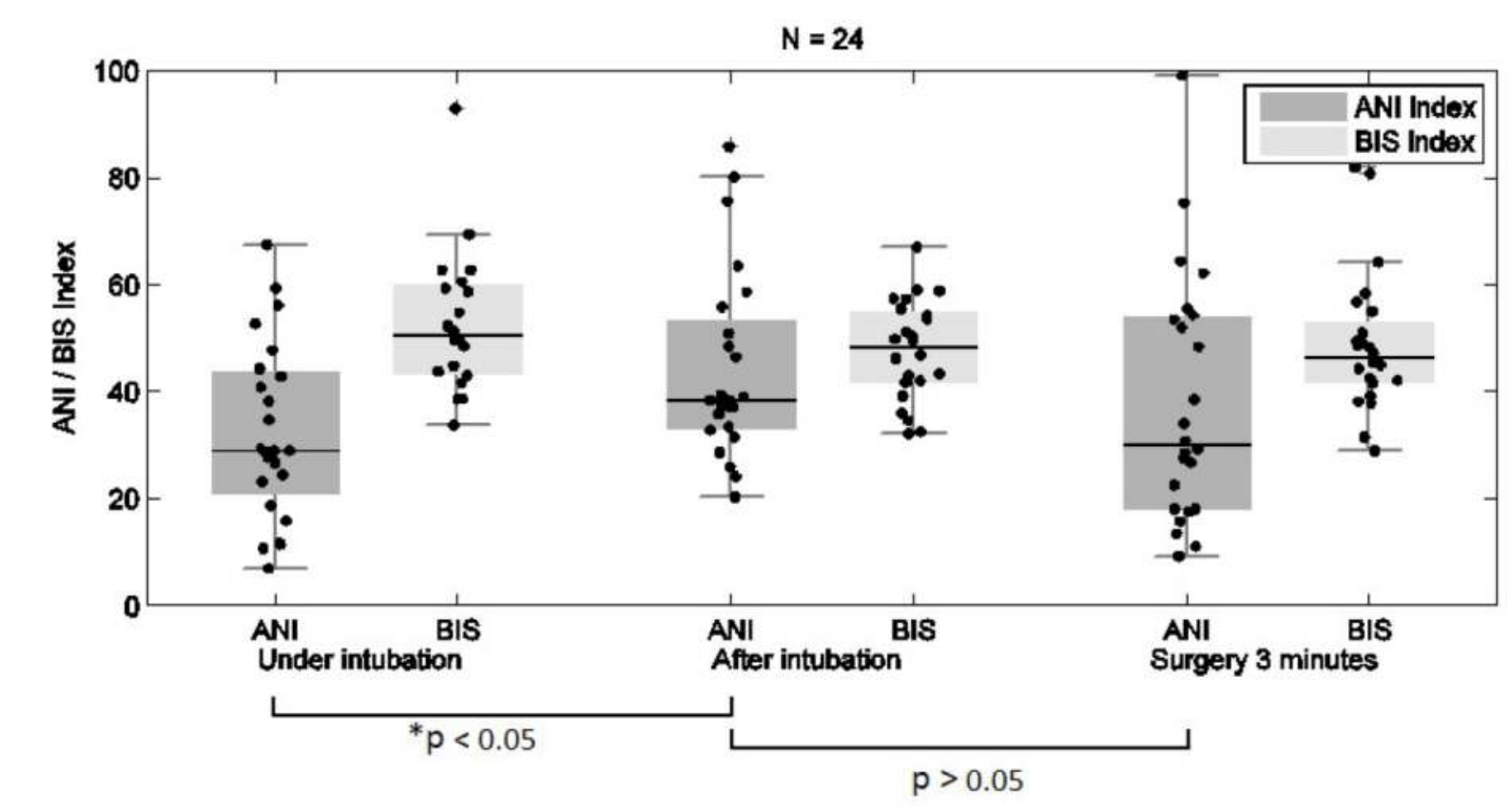

Figure 3. ANI/BIS Index in IVG Group

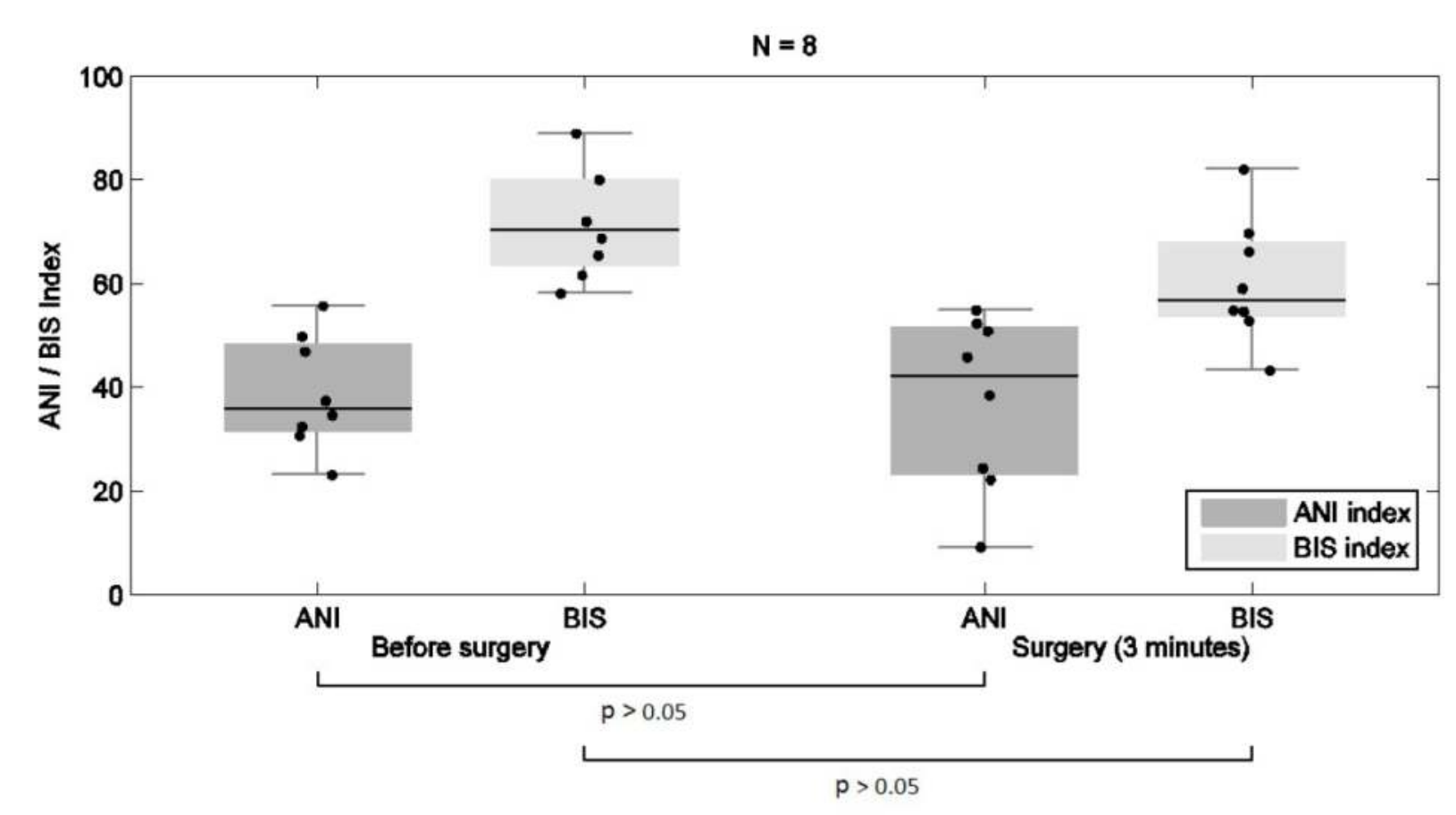

